\title{
Additive envelopes of continuous functions
}

\author{
Bruno H. Strulovici ${ }^{\text {a }}$, Thomas A. Weber ${ }^{\mathrm{b}, *}$ \\ a Department of Economics, Northwestern University, 2001 Sheridan Road, Evanston, IL 60208-2600, United States \\ ${ }^{\mathrm{b}}$ Department of Management Science and Engineering, 442 Terman Engineering Center, Stanford University, Stanford, CA 94305-4026, United States
}

\section{A R T I C L E I N F O}

\section{Article history:}

Received 20 October 2009

Accepted 26 December 2009

Available online 18 January 2010

Keywords:

Additive functions

Pareto optimality

Robust optimization

Superposition

\begin{abstract}
A B S T R A T
We present an iterative method for constructing additive envelopes of continuous functions on a compact set, with contact at a specified point. For elements of a class of submodular functions we provide closedform expressions for such additive envelopes.
\end{abstract}

(C) 2010 Elsevier B.V. All rights reserved.

\section{Introduction}

In this article, we are concerned with finding a continuous additive envelope of a given continuous function on a compact set $X$, a problem that can be reduced to finding a best additive upper bound on $X$. We further require that the envelope has contact with $f$ at a specified point. This problem has interesting applications in contract theory [7] (cf. Section 5), and in optimal transportation where it arises naturally as the dual of the Monge-Kantorovich minimization problem [8].

In response to Hilbert's 13th problem, Kolmogorov [4] showed that it is possible to represent any continuous function $f$ of $n$ variables as a linear superposition of continuous functions of one variable and addition, i.e., $f\left(x_{1}, \ldots, x_{n}\right)=\sum_{k=1}^{2 n+1} g\left(\sum_{l=1}^{n} \kappa_{l} \phi_{k}\left(x_{l}\right)\right)$ for some appropriate continuous functions $\phi_{1}, \ldots, \phi_{2 n+1}, g$, and constants $\kappa_{1}, \ldots, \kappa_{n}$. If nonlinearities are excluded (i.e., $\left.g(x) \equiv x\right)$ ), as in our case, then one obtains a problem of best approximation via linear superposition, which is difficult for $n \geq 3$ [3]. Our problem differs from the standard version in that our approximation is constrained to be without intersection, and with contact at a given point.

We propose an algorithm for finding an additive envelope of an arbitrary continuous function with contact at a specified point. Given any additive upper bound (i.e., a continuous additive function which lies everywhere above the function whose envelope is being computed and which satisfies the contact

\footnotetext{
* Corresponding author.

E-mail addresses: b-strulovici@northwestern.edu (B.H. Strulovici), webert@stanford.edu (T.A. Weber).
}

condition), the algorithm reduces that bound component-bycomponent, and thus yields an improved additive upper bound. At each such iteration the upper bound tightens (unless an additive envelope has been reached). We provide a starting bound for the algorithm, and show that the limiting bound is an additive continuous function and does exist. We also provide conditions (related to supermodularity) on the function and the contact point, which guarantee that the additive envelope is unique and can be obtained in closed form.

\section{Preliminaries}

For some positive integer $n$, let $f \in C\left(\mathbb{R}^{n}, \mathbb{R}\right)$ be a continuous real-valued function and let $X \subset \mathbb{R}^{n}$ be a nonempty compact set. We use the standard definition of continuity with Euclidean norm, which is automatically satisfied when $X$ is finite. Furthermore, we make the following assumptions.

\section{A1 $0 \in X$.}

A2 $f(0)=0$.

A3 $x=\left(x_{1}, \ldots, x_{n}\right) \in X \Rightarrow \forall i \in\{1, \ldots, n\}:\left(0, \ldots, 0, x_{i}\right.$, $0, \ldots, 0) \in X$.

The first two assumptions are without any loss of generality. Indeed, given any $f \in C\left(\mathbb{R}^{n}, \mathbb{R}\right)$ and any $\hat{x} \in X$ (so that $0 \in Y=$ $X-\hat{x})$ the function $g \in C\left(\mathbb{R}^{n}, \mathbb{R}\right)$ with $g(y)=f(y)-f(\hat{x})$ for all $y=x-\hat{x} \in Y$ is such that $g(0)=0$. The function $g$ and the set $Y$ are simple translations of $f$ and $X$, and they satisfy A1-2. The third assumption guarantees that any point $x \in X \subset \mathbb{R}^{n}$ can be represented as a superposition of coordinate vectors which are also elements of $X$. 
A continuous real-valued function $\varphi \in C(X, \mathbb{R})$ is additive if there exist $n$ functions $\varphi_{1}, \ldots, \varphi_{n}$ in $C(\mathbb{R}, \mathbb{R})$ such that

$\varphi(x)=\varphi_{1}\left(x_{1}\right)+\cdots+\varphi_{n}\left(x_{n}\right)$

for any $x=\left(x_{1}, \ldots, x_{n}\right) \in X$. An additive function may be denoted in terms of its components: $\varphi=\left[\varphi_{i}\right]$.

We consider the problem of finding a continuous real-valued function $\varphi \in C(X, \mathbb{R})$ which has the following properties.

P1 $\varphi$ is additive on $X$ (Additivity).

P2 $f \leq \varphi$ on $X$ (One-Sidedness).

P3 If $\hat{\varphi} \in C(X, \mathbb{R})$ is additive on $X$ and $f \leq \hat{\varphi}$ on $X$, then $\varphi \leq \hat{\varphi}$ on $X$ (Minimality).

P4. $\varphi(0)=0$ (Contact at the origin).

A function $\varphi \in C(X, \mathbb{R})$ that satisfies properties P1-3 is called an additive upper envelope of $f$ (on $X$ ). If $\varphi$ satisfies $\mathrm{P} 1-3$ with $f$ replaced by $-f$, then it is called an additive lower envelope of $f$ (on $X$ ). A tuple of functions $H=(\varphi, \hat{\varphi})$, where $\varphi$ is an additive upper envelope of $f$ and $\hat{\varphi}$ is an additive lower envelope of $f$, is called an additive envelope of $f$. It is clear that in order to determine an additive envelope of $f$ we can restrict attention to the problem of finding an additive upper envelope of $f$. We say that an additive upper envelope of $f$ has contact at the origin if P1-4 are satisfied. An additive (upper/lower) envelope $\varphi$ of $f$ is called weak if instead of $\mathrm{P} 3$ the following weaker property holds.

P3' If $\hat{\varphi} \in C(X, \mathbb{R})$ is additive on $X$ and $f \leq \hat{\varphi}$ on $X$, then $\hat{\varphi} \leq \varphi$ on $X$ implies that $\hat{\varphi}=\varphi$ on $X$ (Weak Minimality).

A function $\varphi$ that satisfies P1-2 and P4 is called an additive upper bound of $f$ (with contact at the origin).

\section{Main result}

We start the construction of an additive upper envelope of $f$ on $X$ with an additive upper bound $\varphi^{0} \in C(X, \mathbb{R})$ of $f$, i.e., a function which satisfies properties P1-2 and P4. Such a function always exists. For example, one may start with

$\varphi^{0}(x)=\sum_{i=1}^{n}\left(\max _{\hat{x}_{-i} \in B_{i}\left(x_{i}\right)}\left\{f\left(x_{i}, \hat{x}_{-i}\right)\right\}\right)$,

for all $x \in X$. Here and in what follows, for any $x \in \mathbb{R}^{n}$ and any $i \in\{1, \ldots, n\}$ we use the convenient notation $x=\left(x_{i}, x_{-i}\right)$ and $B_{i}\left(x_{i}\right)=\left\{x_{-i}:\left(x_{i}, x_{-i}\right) \in X\right.$ and $\left.\left\|x_{-i}\right\|_{\infty} \leq\left|x_{i}\right|\right\}$, where $x_{-i}=\left(x_{1}\right.$, $\left.\ldots, x_{i-1}, x_{i+1}, \ldots, x_{n}\right)$ and $\left\|x_{-i}\right\|_{\infty}=\max \left\{\left|x_{1}\right|, \ldots,\left|x_{i-1}\right|,\left|x_{i+1}\right|\right.$, $\left.\ldots,\left|x_{n}\right|\right\}$. The set $B_{i}\left(x_{i}\right)$ contains all points in $X$ which, in the maximum norm, are located not further away from the origin than the coordinate vector $\left(0, \ldots, 0, x_{i}, 0, \ldots, 0\right)$.

Remark 1. A better (i.e., lower) initial additive upper bound than the one suggested in (1) can be obtained as follows. For any nonempty set $N \subseteq\{1, \ldots, n\}$ define

$\Phi_{N}(x)= \begin{cases}\frac{1}{|N|} \sum_{i \in N}\left(\max _{\hat{x}_{-i} \in B_{i}\left(x_{i}\right)}\left\{f\left(x_{i} e_{i}+\sum_{j \in N \backslash\{i\}} \hat{x}_{j} e_{j}\right)\right.\right. \\ \left.\left.-f\left(\sum_{j \in N \backslash\{i\}} \hat{x}_{j} e_{j}\right)\right\}+\Phi_{N \backslash\{i\}}(x)\right), & \text { if }|N| \geq 2, \\ f\left(0, \ldots, 0, x_{j}, 0, \ldots, 0\right), & \text { if } N=\{j\},\end{cases}$

where $e_{i}=(0, \ldots, 0,1,0, \ldots, 0)$ is the $i$-th vector of a standard Euclidean base in $\mathbb{R}^{n}$. Then

$\hat{\varphi}^{0}(x)=\Phi_{\{1, \ldots, n\}}(x)$

is an additive upper bound of $f$ such that $f \leq \hat{\varphi}^{0} \leq \varphi^{0}$ on $X$. In particular, if $f$ is additive, it is straightforward to verify that $\hat{\varphi}^{0}=f$ on $X$. The intuition for the recursive construction of $\Phi_{N}$, which we provide here without detailed proof, becomes clear by considering the simple case where $n=2$. Then,

$$
\begin{aligned}
\Phi_{\{1,2\}}\left(x_{1}, x_{2}\right)= & \frac{1}{2}\left[\max _{\hat{x}_{2} \in B_{1}\left(x_{1}\right)}\left\{f\left(x_{1}, \hat{x}_{2}\right)-f\left(x_{1}, 0\right)\right\}+f\left(x_{1}, 0\right)\right. \\
& \left.+\max _{\hat{x}_{1} \in B_{2}\left(x_{2}\right)}\left\{f\left(\hat{x}_{1}, x_{2}\right)-f\left(0, x_{2}\right)\right\}+f\left(0, x_{2}\right)\right],
\end{aligned}
$$

for all $x \in X$

In order to satisfy $\mathrm{P} 3$, we would like a procedure for tightening a given additive upper bound $\varphi^{k} \in C(X, \mathbb{R})$ that satisfies P1-2 and $\mathrm{P} 4$, where $k \in\{0,1, \ldots\}$. Specifically, given such a function $\varphi^{k}=\left[\varphi_{i}^{k}\right]$ we now construct an improved additive upper bound $\varphi^{k+1}=\sigma\left(\varphi^{k}\right) \in C(X, \mathbb{R})$ as follows. For a given $i \in\{1, \ldots, n\}$ and $x \in X$, set

$\tilde{\varphi}_{i}\left(x_{i}\right)=\max _{x_{-i}:\left(x_{i}, x_{-i}\right) \in X}\left\{f\left(x_{i}, x_{-i}\right)-\sum_{j \neq i} \varphi_{j}^{k}\left(x_{j}\right)\right\}$.

Then consider $\hat{\varphi}=\left[\varphi_{1}, \ldots, \varphi_{i-1}, \tilde{\varphi}_{i}, \varphi_{i+1}, \ldots, \varphi_{n}\right]$ instead of $\varphi=$ $\left[\varphi_{i}\right]$ and repeat (2) for a different index $i$. Repeating this procedure $n$ times, once for each index $i$, we obtain the function $\varphi^{k+1}=$ $\left[\varphi_{i}^{k+1}\right]=\left[\tilde{\varphi}_{i}\right]=\sigma\left(\varphi^{k}\right) \in C(X, \mathbb{R})$. The continuity of $\varphi^{k+1}$ follows from Berge's maximum theorem in [1].

Lemma 1. For any additive upper bound $\varphi^{k}$ of $f$, the function $\varphi^{k+1}=$ $\sigma\left(\varphi^{k}\right)$ is an improved additive upper bound of $f$ in the sense that $\varphi^{k+1} \leq \varphi^{k}$. More specifically, for any $i \in\{1, \ldots, n\}$ : (i) $\varphi_{i}^{k+1} \leq \varphi_{i}^{k}$ on $X$, and (ii) $f \leq \varphi_{i}^{k+1}+\sum_{j \neq i} \varphi_{j}^{k}$ on $X$.

Proof. We obtain from (2), by virtue of P1-2 and P4 with respect to $\varphi^{k}$, that

$\varphi_{i}^{k} \geq \tilde{\varphi}_{i} \geq f-\sum_{j \neq i} \tilde{\varphi}_{j} \geq f-\sum_{j \neq i} \varphi_{j}^{k}$

on $X$, whence

$\varphi_{i}^{k} \geq \varphi_{i}^{k+1} \geq f-\sum_{j \neq i} \varphi_{j}^{k+1} \geq f-\sum_{j \neq i} \varphi_{j}^{k}$

on $X$, i.e., (i) and (ii) obtain. In addition, $\varphi^{k+1} \in C(X, \mathbb{R})$ is an additive upper bound of $f$, since it satisfies $\mathrm{P} 1-2$ and $\mathrm{P} 4$.

By setting $\varphi^{0}$ as in (1) we can construct a sequence $\left\{\varphi^{k}\right\}_{k=0}^{\infty}$ of successively improved additive upper bounds of $f$, where $\varphi^{k+1}=$ $\sigma\left(\varphi^{k}\right)$ for all $k \geq 0$. Lemma 1 implies that this sequence is monotonically decreasing and by $\mathrm{P} 2$ it is bounded from below by $f$. Hence, since every real decreasing sequence which is bounded from below converges ([6], p. 55), there exists a pointwise limit function $\varphi^{\infty}=\left[\varphi_{i}^{\infty}\right]$ with $\varphi_{i}^{\infty}=\lim _{k \rightarrow \infty} \varphi_{i}^{k}$, which may depend on the function $\varphi^{0}$, since $\varphi^{\infty}=\sigma^{\infty}\left(\varphi^{0}\right)$.

Theorem 1. Given any additive upper bound $\varphi^{0}$ of $f$, the limit $\varphi^{\infty}=$ $\sigma^{\infty}\left(\varphi^{0}\right)$ is in $C(X, \mathbb{R})$, and is a weak additive upper envelope of $f$.

Proof. Let $\varphi^{0}$ be any additive upper bound of $f$ with contact at the origin, and let $\varphi^{\infty}=\sigma^{\infty}\left(\varphi^{0}\right)$ be its pointwise limit. By (2) the limit function $\varphi^{\infty}=\left[\varphi_{i}^{\infty}\right]$ satisfies

$\varphi_{i}^{\infty}\left(x_{i}\right)=\sup _{x_{-i}:\left(x_{i}, x_{-i}\right) \in X}\left\{f\left(x_{i}, x_{-i}\right)-\sum_{j \neq i} \varphi_{j}^{\infty}\left(x_{j}\right)\right\}$

for all $i$. Since $f$ is continuous on the compact set $X$ by hypothesis, it is also uniformly continuous on $X$ : given any $\varepsilon>0$ there exists a real number $\delta(\varepsilon)>0$ such that

$\|x-\hat{x}\| \leq \delta(\varepsilon) \Rightarrow|f(x)-f(\hat{x})| \leq \varepsilon / 2$ 
holds for all $x, \hat{x} \in X$. Thus, as long as $\|x-\hat{x}\|=\|\left(x_{i}, x_{-i}\right)-$ $\left(\hat{x}_{i}, \hat{x}_{-i}\right) \| \leq \delta(\varepsilon)$,

$\varphi_{i}^{\infty}\left(x_{i}\right)-\varphi_{i}^{\infty}\left(\hat{x}_{i}\right) \leq f\left(x_{i}, z_{i}\left(x_{i}\right)\right)-f\left(\hat{x}_{i}, z_{i}\left(x_{i}\right)\right) \leq \varepsilon$,

and

$\varphi_{i}^{\infty}\left(\hat{x}_{i}\right)-\varphi_{i}^{\infty}\left(x_{i}\right) \leq f\left(\hat{x}_{i}, z_{i}\left(\hat{x}_{i}\right)\right)-f\left(x_{i}, z_{i}\left(\hat{x}_{i}\right)\right) \leq \varepsilon$,

where $z_{i}\left(x_{i}\right)=\left(z_{i, 1}\left(x_{i}\right), \ldots, z_{i, i-1}\left(x_{i}\right), z_{i, i+1}\left(x_{i}\right), \ldots, z_{i, n}\left(x_{i}\right)\right)$ is such that $\left(x_{i}, z_{i}\left(x_{i}\right)\right) \in X$, and

$\varphi_{i}^{\infty}\left(x_{i}\right)-\left(f\left(x_{i}, z_{i}\left(x_{i}\right)\right)-\sum_{j \neq i} \varphi_{j}^{\infty}\left(z_{i, j}\left(x_{i}\right)\right)\right)<\varepsilon / 2$.

The last inequality means that $\left(x_{i}, x_{-i}\right)=\left(x_{i}, z_{i}\left(x_{i}\right)\right)$ is sufficiently close to achieving the supremum in (3). Hence, using relations (4) and (5) we obtain that for any $x, \hat{x} \in X$,

$\|x-\hat{x}\| \leq \delta(\varepsilon) \Rightarrow\left|\varphi_{i}^{\infty}\left(x_{i}\right)-\varphi_{i}^{\infty}\left(\hat{x}_{i}\right)\right| \leq \varepsilon$,

so that the function $\varphi^{\infty}=\left[\varphi_{i}^{\infty}\right]$ is uniformly continuous on $X$. Clearly, $\varphi^{\infty}$ is an additive upper envelope of $f$ satisfying P1-2 and P4. In addition, $\varphi^{\infty}$ satisfies the weak-minimality property P3' by construction.

Remark 2. The limit function $\varphi^{\infty}=\sigma^{\infty}\left(\varphi^{0}\right)$ depends on the initial function $\varphi^{0}$. However, with respect to the weak minimality criterion P3' any two limit functions $\varphi^{\infty}=\sigma^{\infty}\left(\varphi^{0}\right)$ and $\hat{\varphi}^{\infty}=$ $\sigma^{\infty}\left(\hat{\varphi}^{0}\right)$ are indistinguishable; that is, if the limit functions are different, one cannot weakly dominate the other.

Remark 3. The weak additive envelopes identified by the limit of the sequence $\left\{\varphi^{0}, \varphi^{1}, \ldots\right\}$ are solutions to the system of Eq. (3) for $i \in\{1, \ldots, n\}$. In contrast to the set of all additive upper bounds of $f$ (with or without contact at the origin), the set of additive envelopes given by the limits of our algorithm is generally not convex. Indeed, given any two solutions $\varphi^{\infty}=\left[\varphi_{i}^{\infty}\right]$ and $\hat{\varphi}^{\infty}=$ $\left[\hat{\varphi}_{i}^{\infty}\right]$ their convex combination in general does not satisfy (3), since the supremum is subadditive.

Remark 4. The construction of the weak upper envelope provided above is somewhat related to the levelling algorithm by [2], which in the limit provides the best approximation of an arbitrary function of two variables by a sum of two functions of one variable.

\section{Some exact upper envelopes}

We now examine a class of submodular functions for which, instead of relying on the outcome of the limit process described in the last section, one can obtain an exact expression for a unique additive upper envelope. Recall that a function $h \in C\left(\mathbb{R}^{n}, \mathbb{R}\right)$ is submodular if for any $x, \hat{x} \in \mathbb{R}^{n}: h(x \wedge \hat{x})+h(x \vee \hat{x}) \leq h(x)+$ $h(\hat{x})$, where $x \wedge \hat{x}=\left(\min \left\{x_{1}, \hat{x}_{1}\right\}, \ldots, \min \left\{x_{n}, \hat{x}_{n}\right\}\right)$ and $x \vee \hat{x}=$ $\left(\max \left\{x_{1}, \hat{x}_{1}\right\}, \ldots, \max \left\{x_{n}, \hat{x}_{n}\right\}\right)$.

Theorem 2. Suppose that $f=h \circ g$, where $h \in C\left(\mathbb{R}^{n}, \mathbb{R}\right)$ with $h(0)=0$ is a submodular function, $g=\left(g_{1}, \ldots, g_{n}\right)$ with $g_{i} \in$ $C\left(\mathbb{R}, \mathbb{R}_{+}\right)$and $g_{i}(0)=0$ for all $i \in\{1, \ldots, n\}$. Then assumptions A1-3 imply that the unique additive upper envelope of $f$ is given by

$\varphi(x)=\left[\varphi_{i}\left(x_{i}\right)\right]=\left[h\left(0, \ldots, 0, g_{i}\left(x_{i}\right), 0, \ldots, 0\right)\right]$

for all $\left(x_{1}, \ldots, x_{n}\right) \in X$.
Proof. Since $f(0)=0$ by A2, $h$ is submodular, and all $g_{i}$ 's vanish at the origin and take only nonnegative values, we have using (6) that

$$
\begin{aligned}
f(x) & =h\left(g_{1}\left(x_{1}\right), \ldots, g_{n}\left(x_{n}\right)\right)+0 \\
& \leq \sum_{i=1}^{n} h\left(0, \ldots, 0, g_{i}\left(x_{i}\right), 0, \ldots, 0\right)=\varphi(x)
\end{aligned}
$$

for all $x \in X$. Hence, $\varphi$ satisfies P1-2 and P4 constituting an additive upper bound of $f$ with contact at the origin. We now show that $\varphi$ also satisfies P3. This holds trivially true if $X=\{0\}$. To obtain a contradiction, we thus assume that there is an $x=\left(x_{1}, \ldots, x_{n}\right) \in$ $X$, different from the origin, such that

$f(x) \leq \hat{\varphi}(x)<\varphi(x)$

for some additive upper envelope $\hat{\varphi}=\left[\hat{\varphi}_{i}\right]$ of $f$ which satisfies P1-2 and P4. Assumption A3 guarantees that $\left(0, \ldots, 0, x_{i}, 0, \ldots, 0\right) \in$ $X$ for all $i$. By P4 it is $\hat{\varphi}_{i}(0)=0$, so that by P1-2 we have

$f\left(0, \ldots, 0, x_{i}, 0, \ldots, 0\right)=h\left(0, \ldots, 0, g_{i}\left(x_{i}\right), 0, \ldots, 0\right) \leq \hat{\varphi}_{i}\left(x_{i}\right)$,

for all $i \in\{1, \ldots, n\}$. As a result, by the definition of $\varphi$ in (6) we obtain

$\varphi(x)=\sum_{i=1}^{n} f\left(0, \ldots, 0, x_{i}, 0, \ldots, 0\right) \leq \sum_{i=1}^{n} \hat{\varphi}_{i}\left(x_{i}\right)=\hat{\varphi}(x)$,

a contradiction to (7).

Remark 5. The validity of Theorem 2 critically depends on assumption $\mathrm{A} 3$, which can be seen by considering a simple example. Let $X=\left\{\left(x_{1}, x_{2}\right): x_{1}=x_{2} \in[0,1]\right\}$ be the diagonal of a two-dimensional unit square and let $f(x)=\max \left\{x_{1}, x_{2}\right\}$ be a submodular function (of the form specified in Theorem 2 ) defined on $\mathbb{R}^{2}$, then assumptions A1-2 are satisfied and (6) yields $\varphi(x)=$ $x_{1}+x_{2}=2 x_{1}>x_{1}=f(x)$ for all nonzero $x=\left(x_{1}, x_{2}\right)$ in $X$. However, this additive upper bound can be strictly improved upon by the additive upper envelope $\varphi=\left[\varphi_{i}\right]$ of $f$ with $\varphi_{1}\left(x_{1}\right)=\lambda x_{1}$ and $\varphi_{2}\left(x_{2}\right)=(1-\lambda) x_{2}$, which for any real constant $\lambda$ coincides with $f$ on $X$.

We now provide an application of Theorem 2, which can be used to determine additive envelopes of functions that take weighted averages as arguments, as is frequently the case in practical situations.

Corollary 1. Suppose that $f=h \circ g$, where $h: \mathbb{R} \rightarrow \mathbb{R}$ with $h(0)=0$ is a concave function, $g=\left[g_{i}\right] \in C\left(\mathbb{R}^{n}, \mathbb{R}_{+}\right)$is additive on $X=\left[0, \bar{x}_{1}\right] \times \cdots \times\left[0, \bar{x}_{n}\right]$ for some $\bar{x}=\left(\bar{x}_{1}, \ldots, \bar{x}_{n}\right) \in \mathbb{R}_{+}^{n}$, and $g_{i}(0)=0$ for all $i \in\{1, \ldots, n\}$. Then, the unique additive upper envelope $\varphi=\left[\varphi_{i}\right]$ of $f$ is given by

$\varphi(x)=\left[\varphi_{1}\left(x_{1}\right), \ldots, \varphi_{n}\left(x_{n}\right)\right]=\left[\left(h \circ g_{1}\right)\left(x_{1}\right), \ldots,\left(h \circ g_{n}\right)\left(x_{n}\right)\right]$

for all $\left(x_{1}, \ldots, x_{n}\right) \in X$.

Proof. Note first that $f$ and $X$ satisfy A1-3. It can easily be verified that the concavity of $h$ implies that $\hat{h}\left(y_{1}, \ldots, y_{n}\right)=h\left(y_{1}+\cdots+y_{n}\right)$ is submodular. Thus, Theorem 2 can be directly applied, since $f(x)=\hat{h}\left(g_{1}(x), \ldots, g_{n}(x)\right)$ on $X$.

Examples. Let $c_{1}, c_{2}, \bar{x}_{1}, \bar{x}_{2}>0$, and $X=\left[0, \bar{x}_{1}\right] \times\left[0, \bar{x}_{2}\right]$. (i) For $f\left(x_{1}, x_{2}\right)=\ln \left(1+c_{1} x_{1}+c_{2} x_{2}\right)$ the additive upper envelope $\varphi=\left[\varphi_{i}\right]$ with contact at the origin is given by $\varphi_{i}(x)=\ln \left(1+c_{i} x_{i}\right)$. (ii) For $f\left(x_{1}, x_{2}\right)=-\left(x_{1}^{c_{1}}+x_{2}^{c_{2}}\right)^{2}$ the additive upper envelope $\varphi=\left[\varphi_{i}\right]$ is given by $\varphi_{i}(x)=-x^{2 c_{i}}$. (iii) For $f\left(x_{1}, x_{2}\right)=\left[c_{1} x_{1}^{\rho}+c_{2} x_{2}^{\rho}\right]^{\frac{1}{\rho}}$, with $\rho>1$, the additive upper envelope $\varphi=\left[\varphi_{i}\right]$ is given by $\varphi_{i}(x)=\left(c_{i}\right)^{\frac{1}{\rho}} x_{i}$. 


\section{Discussion}

We have shown that an additive upper bound $\varphi=\left[\varphi_{i}\right]$ of a continuous function $f$ can satisfy the strong minimality property P3 only in certain special cases. In general, the weakminimality property $\mathrm{P3}^{\prime}$ is the most that can naturally be expected of any additive envelope of $f$. This property can be readily interpreted in terms of weak Pareto dominance: if $\varphi$ is an additive upper envelope of $f$, then it is not possible to decrease one coordinate function of $\left[\varphi_{i}\right]$ without increasing another one. The generic multiplicity of weak additive upper envelopes can be illustrated with the following simple example. Consider $f\left(x_{1}, x_{2}\right)=x_{1} x_{2}$ on $X=[-1,1]^{2}$. Then, any $\varphi \in$ $\left\{\left|x_{1}\right|,\left|x_{2}\right|, x_{1}^{2} / 2+x_{2}^{2} / 2\right\}$ is a weak additive upper envelope of $f$ satisfying $\mathrm{P} 1-2, \mathrm{P}^{\prime}$, and $\mathrm{P} 4$.

Another way to interpret weak minimality is in terms of a solution to a variational problem; for any weak additive upper envelope $\varphi$ of $f$ there exists a probability distribution $F$ defined on $X$, such that $\varphi$ solves

$$
\min _{\varphi \in C(X, \mathbb{R})} \int_{X}(\varphi(x)-f(x)) \mathrm{d} F(x),
$$

subject to P1-2 and P4. This feature links the method in Section 3 to problems of equilibrium construction in economics. For instance, the construction of a minimizing sequence of additive upper bounds of $f$ can be directly applied to an equilibrium construction by [7]. They analyze the construction of a complete set of equilibria in a general contracting problem between multiple principals and multiple agents as discussed by [5]. Lastly, we note that the Eq. (3) in Section 3 constitute necessary optimality conditions for (8).

\section{Acknowledgements}

This research was in part supported by a David Morgenthaler faculty scholarship and a presidential faculty grant at Stanford University.

\section{References}

[1] C. Berge, Topological Spaces, Oliver and Boyd, Edinburgh, UK, 1963. Reprinted by Dover Publications, Mineola, NY, in 1997.

[2] S.P. Diliberto, E.G. Straus, On the approximation of a function of several variables by the sum of functions of fewer variables, Pacific Journal of Mathematics 1 (1951) 195-210.

[3] S.Ya. Khavinson, Best Approximation by Linear Superpositions (Approximate Nomography), American Mathematical Society, Providence, RI, 1997.

[4] A.N. Kolmogorov, On the representation of continuous functions of several variables by superpositions of continuous functions of one variable and addition, Doklady Akademii Nauk SSSR 108 (1957) 953-956. English translation in: American Mathematical Society Translations 28 (1961) 369-373.

[5] A. Prat, A. Rustichini, Games played through agents, Econometrica 71 (4) (2003) 989-1026.

[6] W. Rudin, Principles of Mathematical Analysis, Third Edition, McGraw-Hill, New York, NY, 1976

[7] B.H. Strulovici, T.A. Weber, Constructing efficient equilibria in games played through agents, Working Paper, Department of Management Science and Engineering, Stanford University, Stanford, CA, 2009.

[8] C. Villani, Optimal Transport: Old and New, Springer, New York, NY, 2009. 